Linear and nonlinear Rayleigh-Taylor growth at strongly convergent spherical interfaces

D. S. Clark, M. Tabak

January 4, 2006

Physics of Fluids 
This document was prepared as an account of work sponsored by an agency of the United States Government. Neither the United States Government nor the University of California nor any of their employees, makes any warranty, express or implied, or assumes any legal liability or responsibility for the accuracy, completeness, or usefulness of any information, apparatus, product, or process disclosed, or represents that its use would not infringe privately owned rights. Reference herein to any specific commercial product, process, or service by trade name, trademark, manufacturer, or otherwise, does not necessarily constitute or imply its endorsement, recommendation, or favoring by the United States Government or the University of California. The views and opinions of authors expressed herein do not necessarily state or reflect those of the United States Government or the University of California, and shall not be used for advertising or product endorsement purposes. 


\title{
Linear and nonlinear Rayleigh-Taylor growth at strongly convergent spherical interfaces
}

\author{
Daniel S. Clark* and Max Tabak \\ Lawrence Livermore National Laboratory, University of California, Livermore, California 94550
}

(Dated: January 3, 2006)

\begin{abstract}
Recent attention has focused on the effect of spherical convergence on the nonlinear phase of Rayleigh-Taylor growth. For instability growth on spherically converging interfaces, modifications to the predictions of the Layzer model for the secular growth of a single, nonlinear mode have been reported [D. S. Clark and M. Tabak, Phys. Rev. E 72, 0056308 (2005).]. However, this model is limited in assuming a self-similar background implosion history as well as only addressing growth from a perturbation of already nonlinearly large amplitude. Additionally, only the case of singlemode growth was considered and not the multimode growth of interest in applications. Here, these deficiencies are remedied. First, the connection of the recent nonlinear results including convergence to the well-known results for the linear regime of growth is demonstrated. Second, the applicability of the model to more general implosion histories (i.e., not self-similar) is shown. Finally, to address the case of multimode growth with convergence, the recent nonlinear single mode results are combined with the Haan model formulation for weakly nonlinear multimode growth. Remarkably, convergence in the nonlinear regime is found not to modify substantially the multimode predictions of Haan's original model.
\end{abstract}

PACS numbers: 52.57.Fg,47.20.Ky,47.40.-x

\section{INTRODUCTION}

The many instances of the Rayleigh-Taylor (RT) instability $[1,2]$ have motivated its intensive investigation for more than the past half-century. In its simplest incarnation, the RT instability results from the acceleration of a dense fluid by a less dense fluid or, analogously, the dense fluid being supported by the less dense fluid in the presence of gravity. The linear phase of the instability, during which the individual Fourier modes exponentiate in time, has been thoroughly studied in both theory and experiment and under a variety of driving conditions and in various geometries [3-9]. A fairly complete understanding of this phase of the instability is now believed to be in hand. The nonlinear phase of the instability, during which characteristic bubble and spike structures appear, possibly followed by complicating mixing due to secondary instabilities, has also been extensively studied [10-14]. On account of its inherently greater complexity, however, this phase is generally less well understood and a subject of continuing investigation.

In particular, attention has recently focused on the effects of nonlinearity and converging geometry in combination [15-17], such as occurs at the surface of an imploding inertial confinement fusion (ICF) capsule. The effect of spherical convergence on RT growth in the linear regime has long been appreciated, and its potential to enhance perturbation amplitudes over their planar analogues noted [18-20]. This effect is clearly a deleterious one to the goal of ICF and must therefore be heeded in the design of ICF targets [21]. The most recent theoret-

\footnotetext{
${ }^{*}$ Electronic address:clark90@llnl.gov
}

ical investigations have now suggested that an enhancement effect persists into the nonlinear regime of growth [22]. These results indicate that enhancements over the planar predictions, originally due to Layzer [11], can occur for both the acceleration and deceleration phases of an ICF implosion, and for the growth of RT spikes as much as for RT bubbles. However, these recent nonlinear results remain deficient in several respects.

First, in choosing a Layzer-type model as the starting point to analyze the nonlinear growth regime, the connection to the initial conditions remains obscure. By design, the ICF implosions of interest begin with the majority of unstable modes well within the linear RT growth regime. To be relevant to realistic target designs, a bridging of the more recent nonlinear growth results to the well-known early-time, linear results is then necessary.

Second, the construction of the nonlinear convergent growth formulae in Refs. [17, 22] necessitated assuming a self-similar background implosion of the type initially studied by Kidder [23]. While such implosion histories afford a physically reasonable (and mathematically convenient) model of an ICF implosion (especially during the early acceleration phase of the implosion), realistic ICF implosions are in fact much closer to constant acceleration implosions rather than to self-similarity. This is manifestly the case late in the acceleration phase when the self-similar implosion tends to infinite acceleration. Conveniently, self-similarity appears to be less of a liability for the deceleration or stagnation phase where the pure hydrodynamic motion asymptotes to self-similarity around the time of peak compression. In either case, however, generalizing the recent nonlinear results, or otherwise accessing their applicability, to more general (i.e., non-self-similar) implosion histories is desirable.

Finally, the nonlinear results have so far dealt only 
with the case of single, pure modes, while the applications of interest involve the superposition of many unstable modes growing simultaneously. A direct and simple way of treating this multimode case is to follow the procedure outlined by Haan [24]. That enhanced growth has been predicted for pure, nonlinear modes in the presence of convergence seems especially to motivate combining the recent single mode results with the multimode modeling of the type initiated by Haan. Heretofore, convergence has not been explicitly included in the nonlinear phase of these multimode models, and a dangerous underestimation of the multimode amplitudes might therefore be anticipated. A model combining these effects can be hoped to give a more accurate guide in the design of implosion capsules where strong convergence and weakly nonlinear growth are expected.

The objective of this paper is to remedy these three deficiencies and so bring the recent nonlinear growth results into greater relevancy to ICF target design. This paper is organized as follows. Sec. II briefly reviews the growth history for a single mode (linearly and nonlinearly) in the presence of spherical convergence and demonstrates that these growth regimes may be smoothly bridged together in a standard way. Sec. III demonstrates, via simulations, the applicability of the nonlinear regime results to implosion histories other than those that are self-similar. The mechanics of Haan's multimode saturation model are then summarized in Sec. IV. With this background, Sec. V presents a model combining both the effect of convergence in the nonlinear phase and the presence of many modes. Sec. VI concludes.

\section{SINGLE MODE GROWTH WITH SPHERICAL CONVERGENCE}

Very soon after the publication of Taylor's original results on RT growth at planar interfaces, the linear phase of Rayleigh-Taylor growth on converging interfaces was investigated $[18,19]$. A particularly convenient expression for this linear perturbation amplitude on a spheri- cally imploding surface is that given by Hattori [25]:

$$
\begin{aligned}
\eta_{\operatorname{lin}}(t) & =\eta_{0}(\ell) h(t) G(t) \\
G(t) & \doteq \exp \int_{0}^{t} d t\left|\ell \frac{\ddot{h}(t)}{h(t)}\right|^{1 / 2}
\end{aligned}
$$

Here, $\ell$ is the perturbation mode number corresponding approximately to the ratio of the circumference of the unperturbed shell to the perturbation wave length. $\eta_{0}(\ell)$ is the initial perturbation amplitude for mode $\ell$, and $h(t)$ is the implosion history or scale factor which is normalized to unity at the start of the implosion, i.e., $h(t=0) \equiv 1$. During this linear phase, the perturbation shape on the interface corresponds to a Legendre mode $P_{\ell}(\cos \theta)$. This shape and growth rate are for the special case of rotationally symmetric, i.e., $m=0$, modes. Since these have been shown to be the fastest growing modes linearly and nonlinearly [26], only these most threatening modes are considered here.

Eq. (1a) gives only the time-asymptotic amplitude (i.e., terms decaying exponentially in time are neglected) and is valid only in the large mode number limit $(\ell \gg 1)$. For ICF applications, this latter condition is not a significant constraint. Also, like the nonlinear regime results of Ref. [17], this result is strictly valid only for self-similar implosions. However, given that typical implosion histories do not deviate noticeably from self-similarity until late in time and that Eq. (1a) should only ever be applied in the linear (i.e., early-time) regime, Hattori's result may be taken to apply even to general, non-selfsimilar implosions provided only the appropriate linear growth regime is considered. This approximation is essentially the same as that made by Haan in constructing his multimode model.

In Ref. [22], the nonlinear RT bubble growth rate during the acceleration phase of a spherical implosion was shown to be governed by a pair of coupled, nonlinear ODEs involving the implosion scale factor $h(t)$ and the nonlinear bubble amplitude $a(t)$ :

$$
\begin{aligned}
-\frac{\ell}{2}\left(\frac{R_{0}}{t_{c}}\right)^{2} h^{1-3 \gamma} & =a \ddot{a}+\left(1-\frac{\ell}{2}\right) \dot{a}^{2}+2 \frac{\dot{h}}{h} a \dot{a}+\frac{\ell}{2} \frac{\ddot{h}}{h} a^{2} \\
0 & =\left[\frac{b}{a}-\frac{\ell+1}{4}\right]\left[a \ddot{a}+(1-\ell) \dot{a}^{2}+2 \frac{\dot{h}}{h} a \dot{a}\right]-\left[\frac{\ell^{2}-1}{4}+2(1-\ell) \frac{b}{a}\right] \frac{\dot{a}^{2}}{2}+\frac{b}{a} \frac{\ddot{h}}{h} a^{2} .
\end{aligned}
$$

Here, the bubble curvature $b(t)$ is given in terms of the bubble amplitude by

$$
b(t)=\frac{\ell}{4} \frac{\ell+1}{2 \ell-1} a(t)+c_{0} a^{2 \ell}(t)
$$

where $c_{0}$ is an integration constant set by the initial con- ditions. Over-dots denote time derivatives. $\ell$ is now the nonlinear mode number corresponding to the ratio of the circumference of the unperturbed shell to the nonlinear perturbation wavelength, i.e., the full width of the bubble. Though the mode shape is no longer a Legendre 
mode, the mode numbers $\ell$ for the linear and nonlinear regimes may be taken as equivalent. The constant $t_{c}$ sets the characteristic time of the implosion and is determined by the initial and boundary conditions for the pressure and density. $R_{0}$ denotes the characteristic length scale of the implosion, and $\gamma$ is the usual ratio of specific heats.

While they can easily be integrated numerically, these highly nonlinear equations do not evidently have a simple, analytic solution. In the limiting case $\ell \gg 1$, however, they simplify considerably. First, since $a(t)$ should be a decreasing function of time for a bubble penetrating into the dense shell, the second term in Eq. (3) rapidly becomes negligible compared to the first for sufficiently small values of $c_{0}$. Only the first term in the curvature need then be retained at late times. Additionally, Eqs. (2) decouple in the limit of large $\ell$. Independently, Eq. (2a) reduces to the equation studied by Kidder in the context of isentropic, cumulative implosions, while Eq. (2b) may be solved by a WKB technique. For $\gamma=5 / 3$, Eq. (2a) yields the self-similar implosion history $h(t) \simeq h_{\text {Kidder }} \doteq \sqrt{1-\left(t / t_{c}\right)^{2}}$ independent of the perturbation amplitude and mode number. Separately, the WKB solution of Eq. (2b), treating $h(t)$ as given and slowly varying relative to $a(t)$, is

$$
a(t) \sim c_{ \pm}(\ell)\left\{\frac{1}{h} \exp \left[ \pm \sqrt{\ell} \int_{0}^{t} d t \sqrt{-\ddot{h} / h}\right]\right\}^{-1 / \ell}
$$

with $c_{ \pm}(\ell)$ constants. This analysis includes the effect of compressibility of the fluid but, as for the linear regime formula, Eq. (1a), is again strictly valid only for selfsimilar implosions and for large mode numbers $\ell \gg 1$.

In Eq. (4), the amplitude $a(t)$ measures the distance from the center of the imploding sphere to the tip of the growing RT bubble in the frame moving with the imploding interface. Hence, $1-a(t)$ represents the conventional "height" of the bubble above the unperturbed interface. Lengths in the fixed ("lab") frame can be obtained from the imploding frame simply by multiplying by the scale factor $h(t)$. Note that Eq. (1a) is written in the fixed frame, while Eq. (4) is written in the imploding frame. Also note that, even in the absence of acceleration, Eq. (4) suggests that the bubble amplitude can nonetheless grow due to the time-dependence of $h(t)$. This phenomenon may be interpreted as a nonlinear equivalent of the Bell-Plesset effect.

The derivation of Eqs. (2) relied only on the locality in space of the hydrodynamics equations. As such, they apply equally to the linear as to the nonlinear regimes of growth and should include solutions of the type given in Eq. (1a). However, in neglecting the second term in the bubble curvature, Eq. (3), in order to derive Eq. (4), the connection to the initial, linear phase of growth has effectively been lost. Essentially, the $\ell \gg 1$ solution given by Eq. (4) corresponds to the particular choice of initial conditions with a curvature at $t=0$ commensurate with $c_{0}=0$. On the other hand, the linear regime solution, evolving according to Eq. (1a), corresponds to a curvature of $\frac{1}{4} \ell(\ell+1) \eta_{\operatorname{lin}}(t)$, since its shape is by construction a Legendre mode. Matching this curvature to the nonlinear curvature from Eq. (3) at $t=0$ requires the choice

$$
c_{0}=\frac{\ell}{4} \frac{\ell+1}{2 \ell-1}\left[2 \ell \eta_{0}(\ell)-1\right]\left[1-\eta_{0}(\ell)\right]^{-2 \ell}
$$

for which Eqs. (2) appear intractable. Again, a numerical solution can easily be found, but a succinct analytical approximation remains desirable. Intuitively, it can be expected that the real solution must smoothly match together these initial, linear and time-asymptotic, nonlinear curvatures.

A remedy to the intractability of Eqs. (2) when $c_{0} \neq 0$ is to employ the standard linear-nonlinear matching procedure as cited in Layzer's original analysis and attributed to Fermi[11]. This procedure is simply to follow the growth rate given by the linear regime solution until the perturbation speed $\dot{\eta}_{\text {lin }}(t)$ matches the velocity of an RT bubble given by the nonlinear regime solution. After this time, localized bubbles of light fluid can be assumed to rise into the heavy fluid according to the nonlinear growth rate. For the planar case with a perturbation wavelength $\lambda$ and constant gravity $g$, this matching velocity corresponds to the Layzer velocity $u_{\text {Layzer }} \simeq 0.511 \sqrt{\lambda g}$. The accompanying perturbation amplitude at the time of matching or saturation depends merely on the wavelength of the mode, $\eta_{\text {sat }} \sim \lambda / 2 \pi$.

For the present convergent case, a similar procedure may be followed. Since, asymptotically in time, only the growing solution of Eq. (4) is significant, the nonlinear bubble height (in the fixed frame) may be written as

$$
\eta_{\text {non }}(t)=h(t)\left\{1-c_{+}(\ell)\left[\frac{h(t)}{G(t)}\right]^{1 / \ell}\right\} .
$$

Note that, conveniently, the time dependence in the nonlinear phase is of nearly the same functional form as in the linear case; merely the dependence on the mode number is reciprocated. Matching this nonlinear amplitude and corresponding growth rate to the linear regime values from Eq. (1a) sets the undetermined constant $c_{+}(\ell)$ along with the saturation or matching time

$$
\begin{aligned}
c_{+}(\ell) & =\left.\left[\frac{G(t)}{h(t)}\right]^{1 / \ell}\left[1-\eta_{0}(\ell) G(t)\right]\right|_{t=t_{\mathrm{sat}}(\ell)} \\
& \simeq\left\{\ell \eta_{0}(\ell) h\left[t_{\mathrm{sat}}(\ell)\right]\right\}^{-1 / \ell} \\
t_{\mathrm{sat}}(\ell) & \simeq G^{-1}\left[\frac{1}{\ell \eta_{0}(\ell)}\right] .
\end{aligned}
$$

A uniform approximation bridging the linear and nonlinear bubble amplitudes is then simply

$$
\eta_{\text {uni }}(t)=\eta_{\text {lin }}(t) \Theta\left[t_{\text {sat }}(\ell)-t\right]+\eta_{\text {non }}(t) \Theta\left[t-t_{\text {sat }}(\ell)\right]
$$

with $\eta_{\text {lin }}(t)$ and $\eta_{\text {non }}(t)$ taken from Eqs. (1a) and (6), respectively. $\Theta(x)$ is the unit step function.

Fig. 1 plots this uniform single mode bubble amplitude in the frame of the imploding interface for the representative mode $\ell=80$. The smooth solid line shows the result 


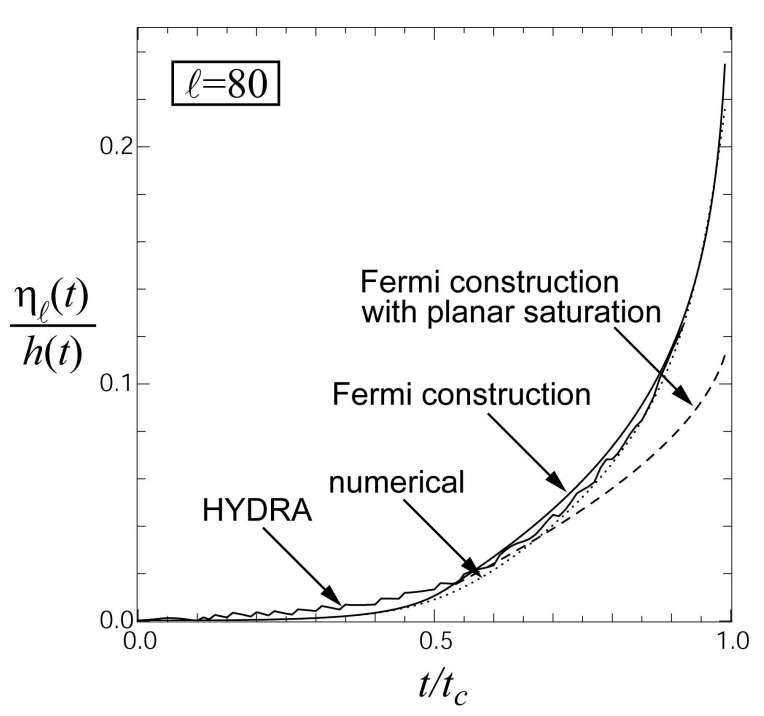

FIG. 1: Linear and nonlinear single mode growth on a spherically converging interface for mode $\ell=80$. Amplitudes are plotted in the frame moving with the interface, and length is normalized to the initial shell radius, i.e., $R_{0} \equiv 1$.

of the Fermi construction, Eq. (7), while the dotted line results from numerically integrating Eqs. (2). For the latter, the constant $c_{0}$ was set to the value given in Eq. (5) so as to match the linear growth in Eq. (1a). The saturation time for these parameters is $t_{\text {sat }}(\ell) \simeq 0.51 t_{c}$, as is evident in the figure. Also plotted with a solid line is the bubble height measured from a two-dimensional (2D) numerical simulation run with the HYDRA code [27]. This simulation was run similarly to those described in Ref. [22], except that an initial perturbation amplitude of $\eta_{0}(\ell) \simeq 0.01 / \ell \simeq 0.002 \lambda$ was used, i.e., well within the linear growth regime. Also, to capture accurately the linear phase of growth, the simulation was run initially in a Lagrangian mode followed by arbitrary Lagrangian Eulerian (ALE) relaxation of the mesh after $t=0.1 t_{c}$. The jitter in the simulation results is due to the ALE relaxation of the mesh and suggests the accuracy threshold of the simulation. Here and below, the unperturbed radius of the sphere has been normalized to unity at $t=0$. Corresponding to the constraint implied in Eqs. (1a) and (4), a self-similar background $h(t)$ is also used.

The close agreement between these three curves indicates that the Fermi construction is in fact a very good approximation to the result of following in detail the evolution of the bubble amplitude (and curvature) in the transition from linear to nonlinear growth. The utility of the Fermi construction in providing a quite simple but equally accurate analytical approximation to a result otherwise obtainable only numerically or through simulations is obvious. By contrast, the dashed line plots the mode amplitude based on the Fermi construction but using Layzer's (planar) saturation velocity, $u_{\text {Layzer }}$ (including the effect of the changing acceleration and bubble wavelength with convergence). Late in time, a near

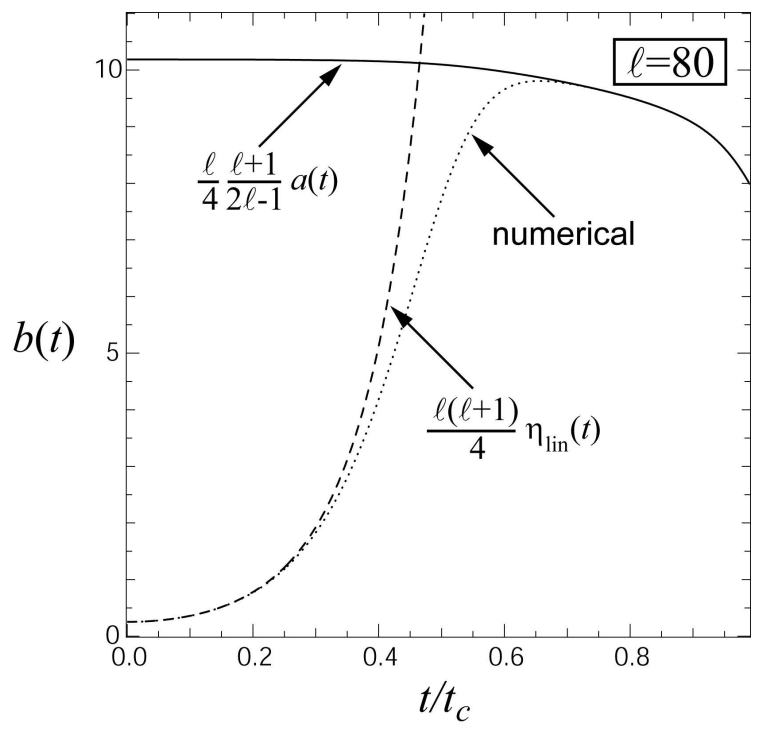

FIG. 2: Linear and nonlinear single mode curvature for mode $\ell=80$ corresponding to the amplitudes shown in Fig. 1 . The dotted curve gives the numerical result, the dashed curve is the curvature corresponding to the linear growth regime, and the solid curve is the time-asymptotic nonlinear curvature (Eq. (3) with $c_{0}=0$ ).

factor of two difference develops between the prediction based on planar saturation and that explicitly including the effect of convergence. Effectively, the results including convergence fail to saturate in the conventional sense but continue growing in a near-exponential fashion. Similar agreement between the numerical, Fermi construction, and simulation results and similar enhancements over the planar analogues are obtained for a range of mode numbers.

Finally, Fig. 2 confirms the expected smooth transition of the bubble curvature from its linear regime value to the time-asymptotic, nonlinear value. The dotted curve gives the value of $b(t)$ computed from the same numerical integration shown in Fig. 1, while the solid curve is the asymptotic curvature value (Eq. (3) with $c_{0}=0$ ). The dashed curve gives the linear regime value. The exponential growth of the numerically computed curvature in the linear regime followed by a smooth asymptoting to the nonlinear value is clearly evident. Note that the curvature which corresponds to the uniformly approximate amplitude given in Eq. (7) would not smoothly follow the numerical solution in Fig. 2, as the amplitude does in Fig. 1, but would jump discontinuously from the linear to the nonlinear regime value at $t=t_{\text {sat }}(\ell)$.

\section{NONLINEAR GROWTH DURING NON-SELF-SIMILAR IMPLOSIONS}

Like the linear regime result of Hattori, the bubble amplitude $a(t)$ in Eq. (4), and hence the uniform approximation in Eq. (7), are strictly valid only for self-similar 


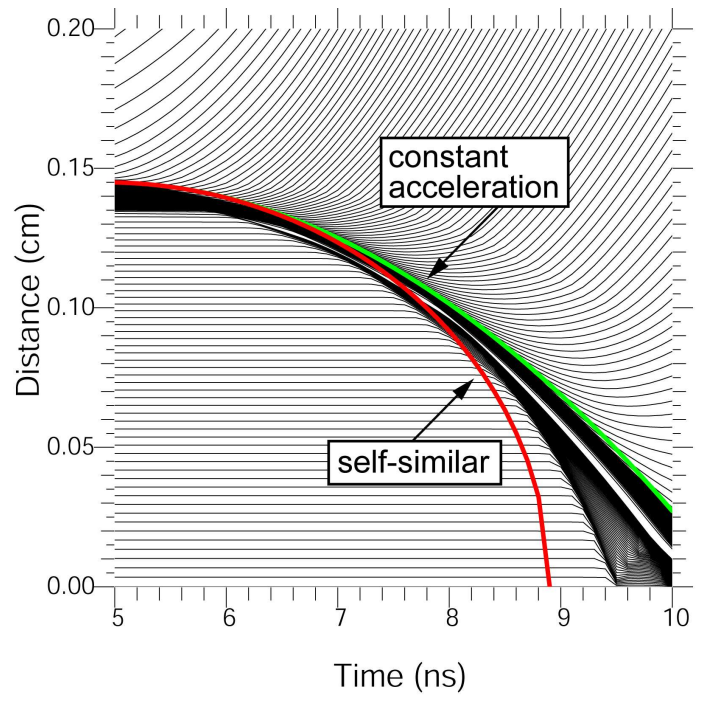

FIG. 3: (Color on-line) Lagrange plot of a typical direct-drive ICF implosion. The green curve marks a trajectory undergoing constant acceleration, and the red curve marks a selfsimilar implosion trajectory with the same initial acceleration. That the dense region of the imploding shell coincides quite closely with the green curve indicates that the acceleration of the shell is nearly constant.

implosions. Although the equation determining $h(t)$ decouples from that determining $a(t)$ when $\ell \gg 1$, the value of $h(t)$ cannot be specified arbitrarily but must be determined self-consistently from Eq. (2a). This constraint of the scale factor $h(t)$ to its self-similar value results from the structure of the nonlinear bubble calculation which both respects the compressibility of the imploding fluid and avoids introducing any singularities in the flow field at the origin.

As already noted, realistic ICF implosion histories are actually better approximated as having constant acceleration rather than being self-similar. Fig. 3 illustrates this with a Lagrange plot of a typical directly driven ICF implosion. The coincidence of the green curve, marking a constantly accelerating trajectory, with the trajectories of the computational zones making up the dense target shell indicates that the outer unstable interface of the shell predominantly experiences a constant acceleration. The red curve, marking a self-similar implosion trajectory with the same initial acceleration, clearly experiences substantially greater acceleration once more than half of the acceleration phase has passed.

Within the structure of the current model, such constant acceleration implosion histories cannot be rigorously treated. However, it may be hoped that the amplitude given by Eq. (4) may still apply in an approximate sense even for a non-self-similar $h(t)$. To the extent that a self-similar implosion history may be approximated by a constant acceleration history merely as a Taylor expansion, i.e., $h(t)=\sqrt{1-t^{2}} \simeq 1-\frac{1}{2} t^{2}$, it can certainly be expected that the bubble amplitude given by Eq. (4), but computed using a constant acceleration $h(t)$, should give a reasonable approximation of the amplitude growth under a constant acceleration drive. This approach is certainly valid at early times when the self-similar and constant acceleration scale factors are indistinguishable on the scale of interest. That this approach remains valid over longer times, when the convergence is strong and errors may accumulate nonlinearly, is not clear.

In the absence of a rigorous solution, the validity of Eq. (4) for non-self-similar implosions may at best be verified by comparison with numerical simulations. Neither of these approaches is, in the absolute sense, an exact solution to the problem at hand. Agreement between two results obtained from wholly different approaches (analytical and numerical) would, however, lend confidence to the validity of each result individually. At the least, if the only real objective is a simple and approximate formula from which to estimate the nonlinear bubble height, and Eq. (4) is shown to comport with simulation results for non-self-similar $h(t)$ s, then such a formula is plausibly had simply by inserting a constant acceleration $h(t)$ into Eq. (4).

Inserting the constant acceleration history $h(t)=1-$ $\frac{1}{2} t^{2}$ into Eq. (4) and choosing the constants $c_{ \pm}(\ell)$ so that $\dot{a}(t=0)=0$ leads directly to

$$
a(t) \sim\left\{\frac{1}{h(t)} \cosh \left[\sqrt{2 \ell} \sin ^{-1} \frac{t}{\sqrt{2}}\right]\right\}^{-1 / \ell} .
$$

This result should be compared with Eq. (10) of Ref. [22]. The predictions of this formula are compared to the bubble heights measured from 2-D HYDRA simulations in Fig. 4. Results for modes $\ell=20,40$, and 80 are shown. Again, these simulations were run similarly to those described in Ref. [22] except that now the pressure source driving the implosion was adjusted to give an implosion history of $h(t)=1-\frac{1}{2} t^{2}$ when the simulation was run in one dimension, i.e., without the perturbation. Note that, by the end of the simulations $\left(t / t_{c} \simeq 1.3\right)$, the shell has converged by more than a factor of $\sim 10(h(t)<0.1)$. The initial radius of the imploding sphere and inward acceleration of the implosion have been normalized to unity. Hence, time is effectively measured in units of the reciprocal square root of the acceleration.

Even though the background implosion is not selfsimilar, the agreement appears to be good between the simulation and WKB results for each mode number. For comparison, the dashed line gives the analogous bubble height from the Layzer model including the effect of the changing bubble wavelength (radius) with convergence. The bubble can be seen to decelerate over the course of the implosion due to the progressive decrease in the bubble wavelength. While the absolute growth driven by constant acceleration is somewhat less extreme than in the case of a self-similar implosion (cf. Fig. 4 of Ref. [22]), the amplitude is still substantially greater than that predicted by the Layzer formula. Note also that, though the WKB result was derived in the limit of large $\ell$, Eq. (4) appears to remain valid even for the relatively modest mode number $\ell=20$. 


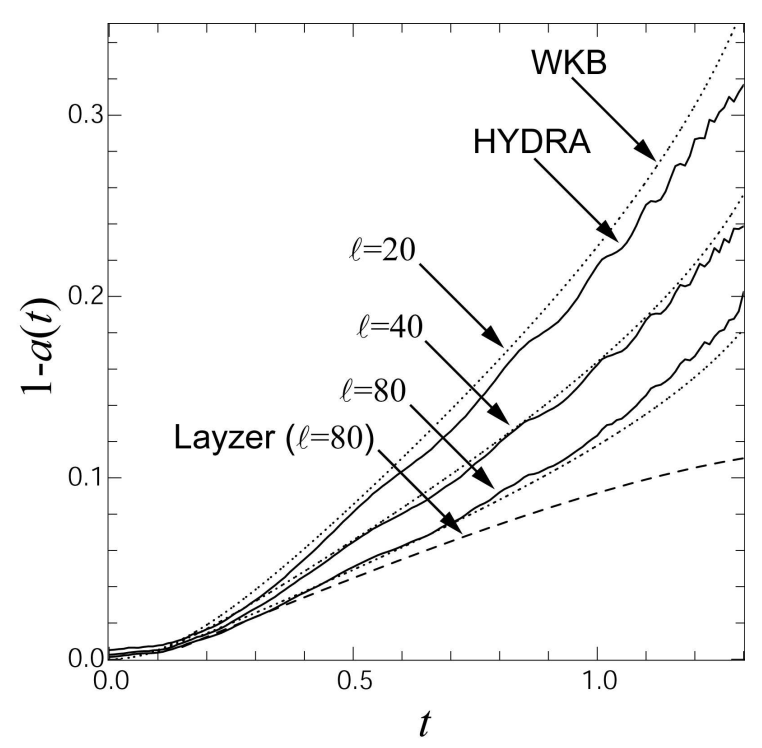

FIG. 4: Comparison between normalized bubble heights from the WKB solution, Eq. (4), (dotted line) and 2-D HYDRA simulations (solid line) for a constant acceleration implosion history and mode numbers $\ell=20,40$, and 80 . The result of the Layzer formula for mode $\ell=80$ is also shown as the dashed line. Amplitudes are plotted in the frame moving with the interface, length is normalized to the initial shell radius, and time is normalized to the square root of the acceleration.

Of course, an infinite variety of non-self-similar implosion histories can be imagined, not merely the constant acceleration case illustrated here. However, this constant acceleration case, as exemplified in Fig. 3, seems sufficiently generic that the good agreement displayed in Fig. 4 should give reasonable confidence in applying the WKB results to a range of similar, if not perfectly constant, acceleration histories. Above all, the essential point remains that the bubble growth with convergence can substantially exceed that predicted by the Layzer model and that this amplitude enhancement may reasonably be approximated with Eq. (4). Finally, extrapolating from these results, a uniformly approximate bubble amplitude could also be constructed for the constant acceleration case following the methods of Sec. II.

\section{HAAN'S MULTIMODE MODEL FOR WEAKLY NONLINEAR GROWTH}

The proceeding discussion has dealt only with the idealized case of growth of a single, pure mode. In ICF and other applications, unstable interfaces will always have some finite and random initial roughness, however, so that growth in the multimode regime will almost invariably be encountered.

The extraordinarily complex interactions of multiple modes in the transition from linear to nonlinear growth make a direct, theoretical treatment of the multimode case extremely difficult. Consequently, approximate, physically-motivated models have been proposed to describe this phase. Broadly, these approximate models fall into the classes of strongly nonlinear bubble merger (or bubble competition) models, as originated by the work of Sharp and Wheeler [28, 29], or weakly nonlinear closure models of the type originated by Haan [24]. In the context of ICF research, models of the type inspired by Haan have proven highly useful in the design of implosion targets, e.g., Ref. [30-34]. Despite the considerable advances in computing power and in numerical techniques, the direct numerical simulation of multimode nonlinear RT growth on an imploding capsule remains a tedious endeavor, and one fraught with uncertainty. Even in the most sophisticated and spatially resolved simulations, numerical diffusion and the effects of extensive grid remapping compete with the instability growth being modeled to an often uncertain degree. Quasi-analytic models, such as Haan's model, hence provide both a guide in assessing the approximate behavior of a target design before detailed simulations are undertaken as well as a rough estimate against which to benchmark those simulation.

The essential ingredient of Haan's multimode saturation model is to include the contribution of neighboring modes to the saturation amplitude of any given mode. In the single mode case discussed above, a mode saturates at an amplitude comparable to some fraction of its wavelength, $\eta_{\text {sat }} \sim \lambda / 2 \pi$, or $\eta_{\text {sat }} \sim h(t) / \ell$ in the spherical case. Haan's model recognizes that, in the presence of a spectrum of initial modes, this nonlinear saturation amplitude may be reached by a coherent sum of modes of comparable wavelengths long before any single mode reaches its saturation amplitude in isolation. By assuming random phasing between neighboring modes, a simple argument leads to a saturation amplitude of $S(\ell)=\nu h(t) / \ell^{2}$ for any given mode in the presence of many modes, i.e., the saturation amplitude is reduced by a factor of $\nu / \ell$ from the single mode value $h(t) / \ell$ on account of the contributions of neighboring modes. The parameter $\nu$ characterizes the spectral extent over which the modes sum coherently and is adjusted to fit multimode experimental data. A value of $\nu \sim 4.0$ is generally considered suitable [35].

After introducing this reduced saturation amplitude, the dynamics of each mode in Haan's model is assumed to follow the single mode Fermi construction as outlined above. The transition from linear to nonlinear, secular growth merely occurs at this much reduced multimode saturation amplitude. Specifically, for any given linear growth amplitude $\eta_{\text {lin }}(t)$, Haan defines the corresponding nonlinear growth amplitude and saturation time to be

$$
\begin{aligned}
\eta_{\text {non }}(t) & =S(\ell)\left\{1+\ln \left[\frac{\eta_{\text {lin }}(t)}{S(\ell)}\right]\right\} \\
t_{\text {sat }}(\ell) & \doteq\left\{t \mid \eta_{\text {lin }}(t)=S(\ell)\right\}
\end{aligned}
$$

Other authors have demonstrated good agreement between 2-D planar simulations and Haan's model, or ex- 
tensions of his model [36, 37]. Favorable agreement with planar experiments has also been reported [38].

Haan's construction of the nonlinear amplitude as the logarithm of the linear amplitude (a construction which he attributes originally to the work of Crowley [39]) is motivated by the single mode solution at a classical, planar interface. For this case, the linear growth is purely exponential in time such that Eq. (9a) yields the linear growth with time to be expected from the Layzer model, albeit with the much reduced bubble velocity corresponding to the lower saturation amplitude. For the spherically convergent case, Haan employs Hattori's Eq. (1a) in the linear regime and the corresponding nonlinear amplitude is calculated from Eq. (9a). In practice, numerical simulations are also often used to extract the linear regime single mode growth factors, i.e., $h(t) G(t)$ from Eq. (1a), by starting from artificially low initial perturbation amplitudes. The linear RT amplitude is then obtained simply by multiplying by the actual initial perturbation amplitude $\eta_{0}(\ell)$, and the nonlinear amplitude follows form Eq. (9a). The justification for this nonlinear amplitude in the convergent case is by no more than an extrapolation from the planar problem, however. Whether this is the correct form at a converging interface remains an open question, especially when extrapolations based on $u_{\text {Layzer }}$ have been shown to underestimate substantially the single mode amplitude, as in Fig. 1.

\section{MULTIMODE NONLINEAR GROWTH INCLUDING THE EFFECT OF CONVERGENCE}

The question of the validity of Eq. (9a) with convergence may be resolved by reformulating Haan's model to include explicitly the nonlinear convergent growth formula from Sec. II. To do so, Haan's formulation of the nonlinear amplitude in terms of a logarithm of the linear amplitude must be replaced by directly matching together the linear and nonlinear growth formulae, as in Sec. II, but at the reduced, multimode saturation amplitude $S(\ell)$. This new matching amplitude necessitates introducing a second constant into the nonlinear growth formula, Eq. (6), which represents the radial location of the bubble apex at the time of saturation, i.e.,

$$
\eta_{\text {non }}(t)=h(t)\left\{\text { const. }(\ell)-c_{+}(\ell)\left[\frac{h(t)}{G(t)}\right]^{1 / \ell}\right\} .
$$

As in the single mode case, matching this nonlinear mode amplitude and growth rate to the linear amplitude and growth rate at the given saturation amplitude $S(\ell)$ de-

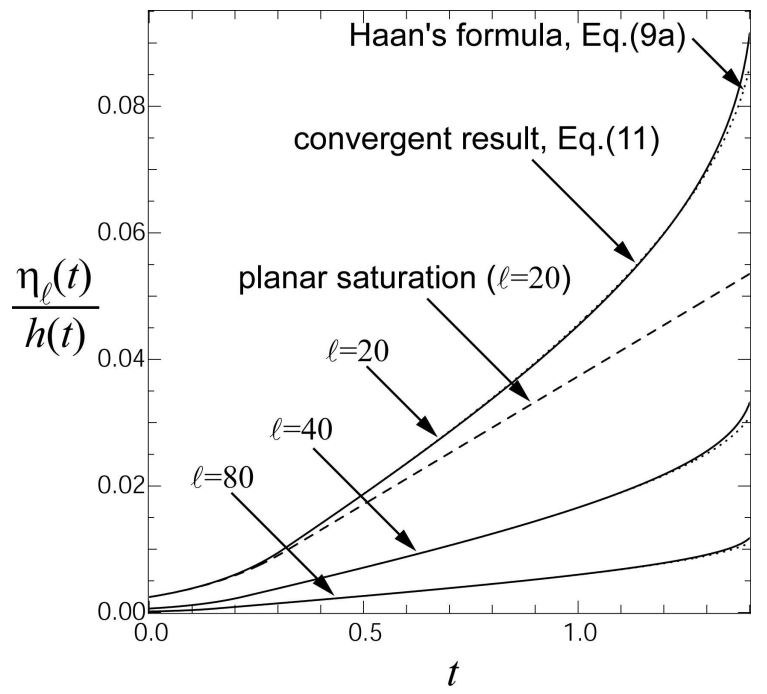

FIG. 5: Linear and nonlinear multimode amplitudes for $\ell=20,40$, and 80 plotted in the frame moving with the imploding interface. The solid curves give the results of Eq. (11), while the dotted curves (only barely distinguishable from the solid curves at late times) are the results of Haan's formula, Eq. (9a). The dashed curve gives the result that would be predicted by the planar saturation rule for $\ell=20$. A nonself-similar drive $h(t)=1-\frac{1}{2} t^{2}$ is used in calculating each curve, and a representative initial perturbation spectrum of $\eta_{0}(\ell)=0.1 /\left(1+0.1 \ell^{2}\right)$ has been chosen. Note that, in comparing the single mode growth history in Fig. 1 to the multimode result, the earlier transition to nonlinear growth in the presence of many modes results in a much reduced nonlinear multimode amplitude relative to the single mode counterpart.

termines the two constants as well as the saturation time

$$
\begin{aligned}
\text { const. }(\ell) & =\left.\frac{\nu}{\ell}\left\{\frac{1}{\ell}+\left[1-\frac{d \ln h(t)}{d \ln G(t)}\right]^{-1}\right\}\right|_{t=t_{\mathrm{sat}}(\ell)} \\
c_{+}(\ell) & =\left.\frac{\nu}{\ell}\left[\frac{\ell^{2}}{\nu} \eta_{0}(\ell) h(t)\right]^{-1 / \ell}\left[1-\frac{d \ln h(t)}{d \ln G(t)}\right]^{-1}\right|_{t=t_{\mathrm{sat}}(\ell)} \\
t_{\mathrm{sat}}(\ell) & \simeq G^{-1}\left[\frac{\nu}{\ell^{2} \eta_{0}(\ell)}\right] .
\end{aligned}
$$

The multimode uniformly approximate bubble amplitude is then, just as in Eq. (7),

$$
\eta_{\text {uni }}(t)=\eta_{\text {lin }}(t) \Theta\left[t_{\text {sat }}(\ell)-t\right]+\eta_{\text {non }}(t) \Theta\left[t-t_{\text {sat }}(\ell)\right]
$$

but now with $\eta_{\text {non }}(t)$ given by Eq. (10) and $t_{\text {sat }}(\ell)$ by the multimode saturation time.

Fig. 5 plots the resulting amplitudes for modes $\ell=20$, 40, and 80 for the multimode convergent case, Eq. (11). For comparison, the results of Haan's formula, Eq. (9a) using Hattori's Eq. (1a), are shown as the dotted lines. A non-self-similar drive $h(t)=1-\frac{1}{2} t^{2}$ has been used in calculating each curve, and a representative initial perturbation spectrum of $\eta_{0}(\ell)=0.1 /\left(1+0.1 \ell^{2}\right)$ has been chosen. Although they have been reached by seemingly very 
different approaches, the results of Eqs. (9a) and (11) are virtually indistinguishable in the figure. The congruence of these curves should not, however, be interpreted as indication that convergence does not modify the nonlinear phase of growth in the multimode case. By contrast, the dashed line gives the result that would be predicted by the planar saturation rule for $\ell=20$, i.e., simply extrapolating the bubble amplitude as growing at the velocity established at the time of saturation. This amplitude is clearly much less than that from Eq. (11) or Haan's result. Instead, Fig. 5 should be taken as confirmation that the logarithm construction proposed by Haan for the nonlinear amplitude is in fact the appropriate procedure to follow in the convergent case, just as it is in the planar case.

The agreement evidenced by Fig. 5 may be seen analytically by noting the identity $x^{\varepsilon} \sim 1+\varepsilon \ln x$ for $\varepsilon \rightarrow 0$ and $x>0$. Recalling that $\ell \gg 1$ is assumed here throughout, this approximation can be seen to transform the convergent nonlinear multimode result, Eq. (10), into Haan's formula, Eq. (9a), up to logarithmic corrections. That this result is possible hinges on the similarity in the time dependence between Eqs. (1a) and (6) already remarked in Sec. II. In effect, the form chosen by Haan for the nonlinear regime, based simply on an extrapolation of the planar results, coupled with Hattori's linear regime results including convergence, conveniently approximates the results of a calculation explicitly including the effect of convergence for both the linear and nonlinear regimes. While the validity of the logarithm construction to other than planar growth might have been anticipated, the results of this section give a more rigorous justification of this approximation. Further, based on the generality of this identity, it should also be noted that this result applies independently of the acceleration history $h(t)$, i.e., is valid for self-similar and constant acceleration histories alike provided the condition $\ell \gg 1$ is satisfied.

The results presented in this section should lend confidence to the estimates of RT growth made using Haan's model. Of course, it must also be borne in mind that these results are valid only in so far as the underlying analysis in Haan's model, and particularly the saturation amplitude $S(\ell)$, are a valid representation of the nonlinear, multimode development of the RT instability. This has proven a contentious issue in the past, especially in the model's application of a Fourier mode decomposition to the nonlinear growth regime. Nevertheless, on account of its broad usage in the ICF design context, assessing the impact on Haan's model made by the recent results on nonlinearity and convergence is certainly warranted.

\section{CONCLUSIONS}

In summary, recent results on the effect of spherical convergence on the nonlinear phase of Rayleigh-Taylor growth have been generalized in three important respects. Each of these generalization brings these results into greater relevance for ICF target design.

First, the connection of the nonlinear phase of growth in converging geometry to the well-known linear growth phase has been demonstrated. As a result, a fairly simple, uniformly valid approximation for the bubble amplitude in the presence of convergence is found. The crucial factor in this connection is the evolution of the perturbation curvature from the linear regime value to the time-asymptotic, nonlinear regime value.

Second, the previous nonlinear regime results, which assumed a self-similar implosion history, were shown to remain valid even for the more typical non-self-similar, constant acceleration implosions. Since self-similarity is an intrinsic ingredient of the nonlinear model, its validity for other implosion histories cannot be rigorously demonstrated and could only be suggested by comparison with numerical simulations.

Lastly, the previous results for single mode nonlinear RT growth with convergences have been combined with the multimode model of Haan to develop a multimode nonlinear RT model which correctly incorporates the effect of convergence. Despite substantially enhancing single mode nonlinear growth, explicitly including convergence in the nonlinear phase is found only slightly to modify the multimode nonlinear amplitude from the predictions of Haan's original formula. This is due to the particular form chosen by Haan in constructing his multimode model. In fact, in the limit of large mode numbers, explicitly including convergence for the nonlinear regime leads only to logarithmic corrections to Haan's original result. While a substantial underestimation of bubble growth is suggested by the discrepancy shown in Fig. 1, Haan's formula, Eq. (9a), effectively already captures these effects of nonlinearity and convergence in combination. This last result should be of particular interest in connection with the many RT growth predictions made using Haan's model.

The possible generalizations of the results presented in Ref. [22] have not been fully exhausted in this paper. Foremost, only the acceleration phase of RT bubble growth has been addressed here. The cases of deceleration phase growth and the growth of RT spikes, also outlined for the single mode, nonlinear case in Ref. [22], could likewise be treated similarly to the analysis given here for acceleration phase bubbles. Ablation of the unstable interface is also a critical effect in ICF and therefore deserves to be included in the analysis of both the linear and nonlinear regimes. Finally, the extension of the present spherical results to cylindrical interfaces is obvious and should be straightforward.

\section{Acknowledgments}

This work was performed under the auspices of the U. S. Department of Energy by the University of California, Lawrence Livermore National Laboratory under Contract No. W-7405-Eng-48. 
[1] Lord Rayleigh, Scientific Papers (Cambridge Univ. Press, Cambridge, 1900).

[2] G. I. Taylor, Proc. R. Soc. London. Ser. A 201, 192 (1950).

[3] S. Chandrasekhar, Hydrodynamic and Hydromagnetic Stability (Oxford Univ. Press, Oxford, 1961).

[4] S. E. Bodner, Phys. Rev. Lett. 33, 761 (1974).

[5] H. Takabe, K. Mima, L. Montierth, and R. L. Morse, Phys. Fluids 28, 3676 (1985).

[6] R. Betti, V. N. Goncharov, R. L. McCrory, P. Sorotokin, and C. P. Verdon, Phys. Plasmas 3, 2122 (1996).

[7] W. W. Hsing and N. M. Hoffman, Phys. Rev. Lett. 78, 3876 (1997).

[8] M. M. Marinak, S. G. Glendinning, R. J. Wallace, B. A. Remington, K. S. Budil, S. W. Haan, R. E. Tipton, and J. D. Kilkenny, Phys. Rev. Lett. 80, 4426 (1998).

[9] S. G. Glendinning, J. Colvin, S. Haan, et al., Phys. Plasmas 7, 2033 (2000).

[10] R. M. Davies and G. I. Taylor, Proc. R. Soc. London. Ser. A 200, 375 (1950).

[11] D. Layzer, Astrophysical. J. 122, 1 (1955).

[12] K. O. Mikaelian, Phys. Rev. Lett. 80, 508 (1998).

[13] Q. Zhang, Phys. Rev. Lett. 81, 3391 (1998).

[14] V. N. Goncharov, Phys. Rev. Lett. 88, 134502 (2002).

[15] S. Atzeni, A. Schiavi, and M. Temporal, Plasma Phys. Controlled Fusion 46, B111 (2004).

[16] V. N. Goncharov and D. Li, Phys. Rev. E 71, 046306 (2005).

[17] D. S. Clark and M. Tabak, Phys. Rev. E 71, 055302 (2005).

[18] G. I. Bell, Taylor instability on cylinders and spheres in the small amplitude approximation, Los Alamos National Laboratory report LA-1321 (1951).

[19] M. S. Plesset, J. Appl. Phys. 25, 96 (1954).

[20] R. Epstein, Phys. Plasmas 11, 5114 (2004).

[21] P. Amendt, J. D. Colvin, J. D. Ramshaw, H. F. Robey, and O. L. Landen, Phys. Plasmas 10, 820 (2003).
[22] D. S. Clark and M. Tabak, Phys. Rev. E 72, 056308 (2005).

[23] R. E. Kidder, Nucl. Fusion 14, 53 (1974).

[24] S. W. Haan, Phys. Rev. A 39, 5812 (1989).

[25] F. Hattori, H. Takabe, and K. Mima, Phys. Fluids 29, 1719 (1986).

[26] M. M. Marinak, B. A. Remington, S. V. Weber, R. E. Tipton, S. W. Haan, K. S. Budil, O. L. Landen, J. D. Kilkenny, and R. Wallace, Phys. Rev. Lett. 75, 3677 (1995).

[27] M. M. Marinak, G. D. Kerbel, N. A. Gentile, O. Jones, D. Munro, S. Pollaine, T. R. Dittrich, and S. W. Haan, Phys. Plasmas 8, 2275 (2001).

[28] D. H. Sharp, Physica D 12, 3 (1984).

[29] J. Glimm and X. Li, Phys. Fluids 31, 2077 (1988).

[30] S. V. Weber, S. G. Glendinning, D. H. Kalantar, et al., Phys. Plasmas 4, 1978 (1997).

[31] V. N. Goncharov, P. McKenty, S. Skupsky, et al., Phys. Plasmas 7, 5118 (2000).

[32] R. L. McCrory, R. E. Bahr, R. Betti, et al., Nucl. Fusion 41, 1413 (2001).

[33] J. D. Lindl, P. Amendt, R. L. Berger, et al., Phys. Plasmas 11, 339 (2004).

[34] P. A. Holstein, D. Babonneau, C. Bowen, et al., Nucl. Fusion 44, S177 (2004).

[35] M. J. Dunning and S. W. Haan, Phys. Plasmas 2, 1669 (1995).

[36] R. P. J. Town, J. D. Findlay, and A. R. Bell, Lasers Part. Beams 14, 237 (1996).

[37] D. Ofer, U. Alon, D. Shvarts, R. L. McCrory, and C. P. Verdon, Phys. Plasmas 3, 3073 (1996).

[38] V. A. Smalyuk, T. R. Boehly, D. K. Bradley, V. N. Goncharov, J. A. Delettrez, and J. P. Knauer, Phys. Rev. Lett. 81, 5342 (1998).

[39] W. P. Crowley, Lawrence Livermore National Laboratory report No. UCRL-72650 (1970). 